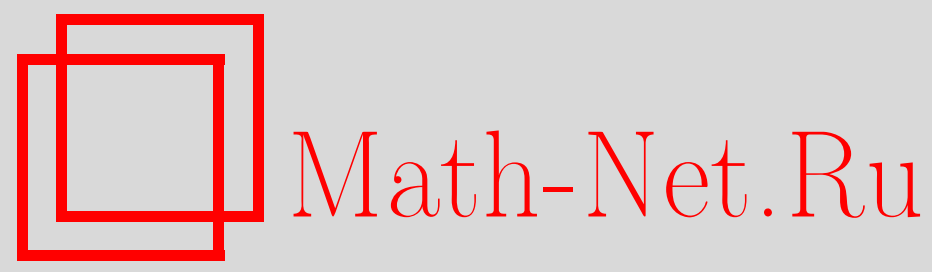

А. Н. Чупрунов, Б. И. Хамдеев, О вероятности исправления ошибок при помехоустойчивом кодировании, если число ошибок случайно, Дискрет. матем., 2010, том 22, выпуск $2,41-50$

DOI: https://doi.org/10.4213/dm1093

Использование Общероссийского математического портала Math-Net.Ru подразумевает, что вы прочитали и согласны с пользовательским соглашением http://www . mathnet.ru/rus/agreement

Параметры загрузки:

IP : 54.164 .48 .24

26 апреля 2023 г., $15: 41: 33$ 


\title{
О вероятности исправления ошибок при помехоустойчивом кодировании, если число ошибок случайно
}

\author{
() 2010 г. А. Н. Чупрунов, Б. И. Хамдеев
}

\begin{abstract}
Рассматривается вероятность $\mathbf{P}(A)$ события $A$, состоящего в том, что при кодировании кодом типа Хэмминга $n$ сообщений, каждое из которых состоит из $N$ блоков, все ошибки будут устранены. При этом предполагается, что $i$-е сообщение имеет $m_{i}=m_{i}\left(\omega_{1}\right)$ ошибок, $\omega_{1} \in \Omega_{1}$, где $m_{i}-$ независимые одинаково распределенные случайные величины, определенные на вероятностном пространстве $\left(\Omega_{1}, \mathfrak{V}_{1}, \mathbf{P}_{1}\right)$. Вероятность $\mathbf{P}(A)$ определяется в терминах обобщенной схемы размещения. Показано, что если $n, N \rightarrow \infty$ так, что $\alpha=n / N \rightarrow \alpha_{0}<\infty$, то вероятности $\mathbf{P}(A)$ сходятся для почти всех $\omega_{1} \in \Omega_{1}$ к одному и тому же пределу, и найдено значение этого предела.
\end{abstract}

\section{1. Введение}

Рассматривается код, который позволяет исправить не больше одной ошибки типа замещения. Частным случаем такого кода является код Хэмминга (см., например, [1]). Работа посвящена изучению вероятности события $A$, состоящего в том, что в $n$ сообщениях, каждое из которых состоит из $N$ блоков, причем каждый блок кодируется нашим помехоустойчивым кодированием, все ошибки будут исправлены.

Пусть случайная величина $\xi_{i j}$ есть число ошибок в $j$-м блоке $i$-го сообщения, случайная величина $m_{i}$ - число ошибок в $i$-м сообщении. Будем предполагать, что $\xi_{i j}-$ независимые неотрицательные целочисленные случайные величины, распределенные так же, как случайная величина $\xi, m_{i}-$ независимые неотрицательные целочисленные случайные величины, распределенные так же, как случайная величина $m$. Случайные величины $\xi_{i j}$ определены на вероятностном пространстве $(\Omega, \mathfrak{A}, \mathbf{P})$, случайные величины $m_{i}$ определены на вероятностном пространстве $\left(\Omega_{1}, \mathfrak{U}_{1}, \mathbf{P}_{1}\right)$. Тогда зависящее от случайного параметра $\omega_{1} \in \Omega_{1}$ событие

$$
A=A\left(\omega_{1}\right)=\bigcap_{i=1}^{n} A_{i}\left(\omega_{1}\right),
$$

где событие

$$
A_{i}=A_{i}\left(\omega_{1}\right)=\left\{\xi_{i 1} \leqslant 1, \xi_{i 2} \leqslant 1, \ldots, \xi_{i N} \leqslant 1 \mid \xi_{i 1}+\ldots+\xi_{i N}=m_{i}\left(\omega_{1}\right)\right\}
$$


состоит в том, что в $i$-м сообщении каждый блок имеет не более одной ошибки при условии, что число ошибок в $i$-м сообщении равно $m_{i}\left(\omega_{1}\right)$. Поэтому

$$
\begin{aligned}
\mathbf{P}(A) & =\mathbf{P}\left(A\left(\omega_{1}\right)\right) \\
& =\left(\mathbf{P}\left\{\xi_{1} \leqslant 1, \xi_{2} \leqslant 1, \ldots, \xi_{N} \leqslant 1 \mid \xi_{1}+\ldots+\xi_{N}=m_{i}\left(\omega_{1}\right)\right\}\right)^{n},
\end{aligned}
$$

где определенные на вероятностном пространстве $(\Omega, \mathfrak{A}, \mathbf{P})$ случайные величины $\xi_{1}, \xi_{2}, \ldots, \xi_{N}$ независимы и распределены так же, как случайная величина $\xi$.

Мы будем предполагать, что распределение случайной величины $\xi$ имеет следующий вид, зависящий от параметра $\theta$. Пусть для последовательности неотрицательных чисел $b_{0}, b_{1}, \ldots$ радиус сходимости $R$ ряда

$$
B(\theta)=\sum_{k=0}^{\infty} \frac{b_{k} \theta^{k}}{k !}
$$

положителен. Тогда случайная величина $\xi=\xi(\theta), 0<\theta<R$, распределена по следующему закону:

$$
\mathbf{P}\{\xi=k\}=\frac{b_{k} \theta^{k}}{k ! B(\theta)}, \quad k=0,1,2, \ldots .
$$

Заметим, что события $A_{i}\left(\omega_{1}\right)$ не зависят от $\theta$. Везде далее мы будем предполагать, что $b_{0}, b_{1}>0$.

Пусть $\alpha=n / N$. Нами показано, что если $\mathbf{E}_{1} m ! \lambda^{-m}<\infty$ для некоторого $0<\lambda<\infty$, To

$$
\mathbf{P}(A) \rightarrow \exp \left(-\frac{\alpha_{0}}{2} \frac{b_{2} b_{0}}{\left(b_{1}\right)^{2}} \mathbf{E} I_{\{m \geqslant 2\}} m(m-1)\right)
$$

почти наверное при $n, N \rightarrow \infty$ так, что $\alpha \rightarrow \alpha_{0}$ (теорема 1 ). Если случайные величины $\xi_{i j}$ имеют пуассоновское распределение (случай $b_{0}=b_{1}=\ldots$ ), то этот результат верен при более слабом условии $\mathbf{E}_{1} m^{2}<\infty$ (теорема 2).

Событие $A_{i}\left(\omega_{1}\right)$ является событием обобщенной схемы размещения: событие $A_{i}\left(\omega_{1}\right)$ состоит в том, что в обобщенной схеме размещения $m_{i}\left(\omega_{1}\right)$ частиц по $N$ ячейкам в каждой ячейке оказалось не более одной частицы. Обобщенная схема размещения введена в [2] (см. также [3]). Случайные величины $\xi_{i}(\theta)$ были введены в [4]. В [4-6] получены предельные теоремы для сумм случайных величин $\xi_{i}(\theta)$. Теорема 1 настоящей работы основана на уточнении пуассоновской предельной теоремы из [4], полученном в лемме 1.

\section{2. Основная теорема}

Рассмотрим вероятность

$$
B(m, N)=\mathbf{P}\left\{\xi_{j} \leqslant 1,1 \leqslant j \leqslant N, \xi_{1}+\xi_{2}+\xi_{3}+\ldots+\xi_{N}=m\right\} .
$$

Легко заметить, что

$$
B(m, N)=\left(\begin{array}{l}
N \\
m
\end{array}\right)\left(\frac{b_{1} \theta}{B(\theta)}\right)^{m}\left(\frac{b_{0}}{B(\theta)}\right)^{N-m}
$$


Следовательно, если $N \theta b_{1} / b_{0} \rightarrow \lambda$ при $N \rightarrow \infty$, то при любом фиксированном $m=0,1, \ldots$

$$
B(m, N) \rightarrow e^{-\lambda} \frac{\lambda^{m}}{m !}
$$

Мы будем предполагать, что $\sum_{i \in \varnothing} a_{i}=0, \prod_{i \in \varnothing} a_{i}=1$. Также мы будем использовать неравенства

$$
\left(\begin{array}{l}
N \\
k
\end{array}\right) \frac{1}{N^{k}} \leqslant \frac{1}{k !}, \quad \frac{b_{0}}{B(\theta)} \leqslant 1 .
$$

В [4] (теорема 2) доказано, что, если $N \theta b_{1} / b_{0} \rightarrow \lambda$ при $N \rightarrow \infty$, то

$$
\mathbf{P}\left\{\xi_{1}+\xi_{2}+\ldots+\xi_{N}=m\right\} \rightarrow e^{-\lambda} \frac{\lambda^{m}}{m !}
$$

для любого фиксированного $m=0,1, \ldots$ Нам потребуется следующее уточнение этого утверждения.

Лемма 1. Пусть

$$
\theta=\theta(N)=\frac{b_{0} \lambda}{b_{1} N}=\frac{C_{1}}{N}, \quad C_{2}=\frac{b_{2} b_{0} \lambda^{2}}{2\left(b_{1}\right)^{2}}
$$

Виберем $N_{0} \in \mathbf{N}$ такое, что

$$
C_{3}=\sum_{i=3}^{\infty} \frac{b_{i}}{i !}\left(\theta\left(N_{0}\right)\right)^{i-3}<\infty
$$

Тогда при $N>N_{0}$ и $m \leqslant N$ справедливы следующие соотношения: для $m=0,1$

$$
\mathbf{P}\left\{\xi_{1}+\ldots+\xi_{N}=m\right\}=B(m, N)
$$

для $m=2$

$$
\mathbf{P}\left\{\xi_{1}+\ldots+\xi_{N}=m\right\}=B(m, N)+\frac{1}{N} \frac{b_{2}\left(C_{1}\right)^{2}}{2 B(\theta)} B(m-2, N-1)
$$

для $m=3$

$$
\mathbf{P}\left\{\xi_{1}+\ldots+\xi_{N}=m\right\}=B(m, N)+\frac{1}{N} \frac{b_{2}\left(C_{1}\right)^{2}}{2 B(\theta)} B(m-2, N-1)+\frac{C}{N^{2}},
$$

где $0 \leqslant C \leqslant C_{4}, C_{4}=\left(C_{1}\right)^{3} e^{\lambda} b_{3} / 6 b_{0} ;$ для $m>3$

$$
\mathbf{P}\left\{\xi_{1}+\ldots+\xi_{N}=m\right\}=B(m, N)+\frac{1}{N} \frac{b_{2}\left(C_{1}\right)^{2}}{2 B(\theta)} B(m-2, N-1)+\frac{C}{N^{2}},
$$

где $0 \leqslant C \leqslant C_{5}$ и $C_{5}=C_{5}(\lambda)=\left(C_{1}\right)^{3} e^{\lambda} b_{3} / 6 b_{0}+\left(C_{3} C_{1}^{3}+\left(C_{2}\right)^{2} / 2\right) e^{C_{2} / N+\lambda}$. 
Доказательство. Введем обозначения

$$
v_{1}=\sum_{j=1}^{N} \xi_{j} I_{\left\{\xi_{j} \leqslant 1\right\}}, \quad v_{2}(k)=\sum_{j=1}^{k} \xi_{j} I_{\left\{\xi_{j} \geqslant 2\right\}}
$$

При $k=N$ мы будем обозначать случайную величину $v_{2}(k)$ через $v_{2}$. Рассмотрим выражение

$$
\mathbf{P}\left\{\xi_{1}+\ldots+\xi_{N}=m\right\}=\sum_{i=0}^{m} \mathbf{P}\left(v_{1}=m-i, v_{2}=i\right) .
$$

Заметим, что

$$
\mathbf{P}\left(v_{1}=m, v_{2}=0\right)=B(m, N), \quad \mathbf{P}\left(v_{1}=m-1, v_{2}=1\right)=0
$$

И

$$
\begin{aligned}
\mathbf{P}\left(v_{1}=m-2, v_{2}=2\right) & =\sum_{j=1}^{N} \mathbf{P}\left(v_{1}=m-2, \xi_{j}=2, \xi_{i}<2, i \neq j\right) \\
& =N \mathbf{P}\left(\sum_{j=1}^{N} \xi_{i} I_{\left\{\xi_{i} \leqslant 1\right\}}=m-2, \xi_{1}=2, \xi_{i}<2,2 \leqslant i \leqslant N\right) \\
& =N \mathbf{P}\left(\xi_{1}=2\right) \mathbf{P}\left(\sum_{j=2}^{N} \xi_{i} I_{\left\{\xi_{i} \leqslant 1\right\}}=m-2, \xi_{i}<2,2 \leqslant i \leqslant N\right) \\
& =N \frac{b_{2} \theta^{2}}{2 B(\theta)} B(m-2, N-1),
\end{aligned}
$$

и так как

$$
\mathbf{P}(\xi=1) \leqslant \frac{\lambda}{N}
$$

справедливы соотношения

$$
\begin{aligned}
\mathbf{P}\left(v_{1}=m-3, v_{2}=3\right) & =\left(\begin{array}{c}
N \\
m-3
\end{array}\right)(\mathbf{P}(\xi=1))^{m-3} \mathbf{P}\left(v_{2}(N-(m-3))=3\right) \\
& \leqslant\left(\begin{array}{c}
N \\
m-3
\end{array}\right) \frac{\lambda^{m-3}}{N^{m-3}}(N-(m-3)) \frac{b_{3}}{6 B(\theta)} \theta^{3} \leqslant \frac{e^{\lambda}}{N^{2}} \frac{b_{3}}{6 b_{0}} C_{1}^{3} .
\end{aligned}
$$

Равенства (9) и (10) влекут (5), равенства (9), (10) и (11) влекут (6), равенства (9), (10), (11) и оценка (12) влекут (7).

Рассмотрим сумму

$$
\sum_{i=4}^{m} \mathbf{P}\left(v_{1}=m-i, v_{2}=i\right)=\sum_{i=4}^{m}\left(\begin{array}{c}
N \\
m-i
\end{array}\right)(\mathbf{P}(\xi=1))^{m-i} \mathbf{P}\left(v_{2}(N-(m-i))=i\right) .
$$

Введем обозначение

$$
v_{3}(k)=\sum_{j=1}^{k} \xi_{j} I_{\left\{\xi_{j} \geqslant 3\right\}}
$$


Так как

$$
\mathbf{P}(\xi=2) \leqslant \frac{C_{2}}{N^{2}}, \quad \mathbf{P}(\xi=2) \leqslant \frac{C_{2}}{N^{2}},
$$

справедливы оценки

$$
\begin{aligned}
\mathbf{P}\left(v_{2}(N-(m-i))=i\right)= & \sum_{k=0}^{[i / 2]}\left(\begin{array}{c}
N-(m-i) \\
k
\end{array}\right)(\mathbf{P}(\xi=2))^{k} \\
& \times \mathbf{P}\left(v_{3}(N-(m-i)-k)=i-2 k\right) \\
\leqslant & \sum_{k=0}^{[i / 2]}\left(\begin{array}{c}
N-(m-i) \\
k
\end{array}\right)\left(\frac{C_{2}}{N^{2}}\right)^{k} \\
& \times \mathbf{P}\left(v_{3}(N-(m-i)-k)=i-2 k\right) \\
\leqslant & \sum_{k=0}^{[i / 2]} \frac{1}{k !}\left(\frac{C_{2}}{N}\right)^{k} \mathbf{P}\left(v_{3}(N-(m-i)-k)=i-2 k\right) .
\end{aligned}
$$

Заметим, что

$$
\mathbf{P}\left(v_{3}(k)=j\right) \leqslant \mathbf{P}\left(v_{3}(k) \geqslant j\right) \leqslant k \mathbf{P}(\xi \geqslant 3) \leqslant \frac{C_{3}}{N^{2}}
$$

при $k \leqslant N$ и $j \geqslant 3$. Отсюда получаем, что

$$
\begin{aligned}
\mathbf{P}\left(v_{2}(N-(m-i))=i\right) & \leqslant \sum_{k=0}^{(i-1) / 2} \frac{\left(C_{2} / N\right)^{k}}{k !} \frac{C_{3} C_{1}^{3}}{N^{2}} \\
& \leqslant \frac{C_{3} C_{1}^{3}}{N^{2}} \exp \left(\frac{C_{2}}{N}\right),
\end{aligned}
$$

если $i$ нечетно, и

$$
\begin{aligned}
\mathbf{P}\left(v_{2}(N-(m-i))=i\right) & \leqslant \sum_{k=0}^{i / 2-1} \frac{\left(C_{2} / N\right)^{k}}{k !} \frac{C_{3} C_{1}^{3}}{N^{2}}+\frac{\left(C_{2} / N\right)^{i / 2}}{(i / 2) !} \\
& \leqslant \frac{C_{3} C_{1}^{3}}{N^{2}} \exp \left(\frac{C_{2}}{N}\right)+\frac{\left(C_{2} / N\right)^{i / 2}}{(i / 2) !},
\end{aligned}
$$

если $i$ четно. Из (14), (15) и (16) вытекает, что

$$
\begin{aligned}
& \sum_{i=4}^{m} \mathbf{P}\left(v_{1}=m-i, v_{2}=i\right) \\
& \leqslant \frac{C_{3} C_{1}^{3}}{N^{2}} e^{C_{2} / N} \sum_{i=4}^{m}\left(\begin{array}{c}
N \\
m-i
\end{array}\right)\left(\frac{\lambda}{N}\right)^{m-i}+\sum_{\substack{4 \leqslant i \leqslant m \\
i \in \mathbf{2}}}\left(\begin{array}{c}
N \\
m-i
\end{array}\right)\left(\frac{\lambda}{N}\right)^{m-i} \frac{\left(C_{2} / N\right)^{i / 2}}{(i / 2) !} \\
& \leqslant \frac{C_{3} C_{1}^{3}}{N^{2}} e^{\lambda+C_{2} / N}+\left(\frac{C_{2}}{N}\right)^{2} \sum_{\substack{4 \leqslant i \leqslant m \\
i \in \mathbf{2}}}\left(\begin{array}{c}
N \\
m-i
\end{array}\right)\left(\frac{\lambda}{N}\right)^{m-i} \frac{\left(C_{2} / N\right)^{i / 2}-2}{(i / 2-2) !} \\
& \leqslant \frac{C_{3} C_{1}^{3}}{N^{2}} e^{C_{2} / N+\lambda}+\frac{\left(C_{2}\right)^{2}}{2 N^{2}} e^{C_{2} / N} \sum_{i=4}^{m}\left(\begin{array}{c}
N \\
m-i
\end{array}\right)\left(\frac{\lambda}{N}\right)^{m-i} \leqslant \frac{1}{N^{2}} e^{C_{2} / N+\lambda}\left(C_{3} C_{1}^{3}+\frac{\left(C_{2}\right)^{2}}{2}\right),
\end{aligned}
$$


где $\mathbf{2 N}$ - множество четных чисел.

Тогда (9), (10), (11), (12) и (17) влекут (8). Доказательство закончено.

Замечание 1. Лемма 1 показывает, что распределение суммы $\xi_{1}+\xi_{2}+\ldots+\xi_{N}=m$ хорошо аппроксимируется биномиальным распределением, которое, согласно пуассоновской предельной теореме, сходится к пуассоновскому распределению. Таким образом, мы получаем новое доказательство теоремы 2 из [4]. Используя лемму 1, можно также дать новое доказательство локальной центральной предельной теоремы из [4]. Для этого достаточно в (8) оценить величины $B(m, N)$ с помощью теоремы Муавра-Лапласа. Из леммы 1 следует, что сходимость в (4) равномерна по $\lambda$, принадлежащим ограниченному множеству.

Мы будем использовать следующее простое следствие усиленного закона больших чисел.

Лемма 2. Пусть $\eta, \eta_{i}, i \in \mathbf{N},-$ такие независимые одинаково распределеннье случайные величины, что $\mathbf{E}|\eta|<\infty$. Тогда

$$
\frac{1}{N} \sum_{i=1}^{n} \eta_{i} \rightarrow \alpha_{0} \mathbf{E} \eta
$$

почти наверное, если $n, N \rightarrow \infty$ так, что $\alpha \rightarrow \alpha_{0}$.

Лемма 2 следует из усиленного закона больших чисел А. Н. Колмогорова, примененного к равенству

$$
\frac{1}{N} \sum_{i=1}^{n} \eta_{i}=\alpha \frac{1}{n} \sum_{i=1}^{n} \eta_{i}
$$

Теорема 1. Пусть $\mathbf{E}_{1} m ! \lambda^{-m}<\infty$ для некоторого $0<\lambda<\infty$. Тогда почти наверное

$$
\mathbf{P}(A) \rightarrow \exp \left(-\frac{\alpha_{0}}{2} \frac{b_{2} b_{0}}{\left(b_{1}\right)^{2}} \mathbf{E} I_{\{m \geqslant 2\}} m(m-1)\right),
$$

если $n, N \rightarrow \infty$ так, что $\alpha \rightarrow \alpha_{0}$.

Доказательство. Пусть

$$
\theta=\theta(N)=\frac{b_{0} \lambda}{b_{1} N} .
$$

Условие $\mathbf{E}_{1} m ! \lambda^{-m}<\infty$ влечет нерапвенство $\mathbf{E}_{1} I_{\{m \geqslant 2\}} m(m-1)<\infty$. Поэтому по лемме 2 найдется $\Omega^{\prime} \subset \Omega_{1}$ такое, что $\mathbf{P}_{1}\left(\Omega^{\prime}\right)=1$ и для всех $\omega_{1} \in \Omega^{\prime}$

$$
\begin{aligned}
& \frac{1}{N} \sum_{i=1}^{n} m\left(\omega_{1}\right) ! \lambda^{-m(\omega)} \rightarrow \alpha_{0} \mathbf{E}_{1} m ! \lambda^{-m} \\
& \frac{1}{N} \sum_{i=1}^{n} I_{\left\{m_{i}\left(\omega_{1}\right) \geqslant 2\right\}} m_{i}\left(\omega_{1}\right)\left(m_{i}\left(\omega_{1}\right)-1\right) \rightarrow \alpha_{0} \mathbf{E}_{1} I_{\{m \geqslant 2\}} m(m-1) \text {, }
\end{aligned}
$$

если $n, N \rightarrow \infty$ так, что $\alpha \rightarrow \alpha_{0}$. 
Пусть $\omega_{1} \in \Omega^{\prime}$. Условия (18) и (19) влекут сходимости

$$
\frac{1}{N} m_{n}\left(\omega_{1}\right) ! \lambda^{-m_{n}\left(\omega_{1}\right)} \rightarrow 0, \quad \frac{m_{n}\left(\omega_{1}\right)\left(m_{n}\left(\omega_{1}\right)-1\right)}{N} \rightarrow 0,
$$

если $n, N \rightarrow \infty$ так, что $\alpha \rightarrow \alpha_{0}$. Поэтому

$$
\frac{m_{n}\left(\omega_{1}\right)}{N} \rightarrow 0
$$

если $n, N \rightarrow \infty$ так, что $\alpha \rightarrow \alpha_{0}$. Следовательно,

$$
\begin{aligned}
\max _{1 \leqslant i \leqslant n} \frac{1}{N} m\left(\omega_{1}\right) ! \lambda^{-m\left(\omega_{1}\right)} e^{-m\left(\omega_{1}\right)\left(m\left(\omega_{1}\right)-1\right) / 2} & \rightarrow 0, \\
\max _{1 \leqslant i \leqslant n} \frac{m_{i}\left(\omega_{1}\right)}{N} & \rightarrow 0, \\
\max _{1 \leqslant i \leqslant n} \frac{m_{i}\left(\omega_{1}\right)\left(m_{i}\left(\omega_{1}\right)-1\right)}{N} & \rightarrow 0,
\end{aligned}
$$

если $n, N \rightarrow \infty$ так, что $\alpha \rightarrow \alpha_{0}$.

Заметим, что

$$
\begin{aligned}
& \frac{B\left(m_{i}\left(\omega_{1}\right)-2, N-1\right)}{B\left(m_{i}\left(\omega_{1}\right), N\right)} \\
& =\frac{(N-1) \ldots\left(N-1-\left(m_{i}\left(\omega_{1}\right)-2\right)+1\right)}{\left(m_{i}\left(\omega_{1}\right)-2\right) !}\left(\frac{b_{1} \theta}{B(\theta)}\right)^{m_{i}\left(\omega_{1}\right)-2}\left(\frac{b_{0}}{B(\theta)}\right)^{N-1-\left(m_{i}\left(\omega_{1}\right)-2\right)} \\
& \quad \times\left(\frac{N(N-1) \ldots\left(N-m_{i}\left(\omega_{1}\right)+1\right)}{m_{i}\left(\omega_{1}\right) !}\left(\frac{b_{1} \theta}{B(\theta)}\right)^{m_{i}\left(\omega_{1}\right)}\left(\frac{b_{0}}{B(\theta)}\right)^{N-m_{i}\left(\omega_{1}\right)}\right)^{-1} \\
& =\frac{m_{i}\left(\omega_{1}\right)\left(m_{i}\left(\omega_{1}\right)-1\right)}{N\left(N-m_{i}\left(\omega_{1}\right)+2\right)} \frac{b_{0} B(\theta)}{\left(b_{1} \theta\right)^{2}}=\frac{m_{i}\left(\omega_{1}\right)\left(m_{i}\left(\omega_{1}\right)-1\right)}{1-m_{i}\left(\omega_{1}\right) / N+2 / N} \frac{b_{0} B(\theta)}{\left(b_{1} C_{1}\right)^{2}} .
\end{aligned}
$$

Поэтому при $m_{i}\left(\omega_{1}\right) \geqslant 2$ по лемме 1 получаем, что

$$
\begin{aligned}
\mathbf{P}\left(A_{i}\left(\omega_{1}\right)\right) & =\frac{B\left(m_{i}\left(\omega_{1}\right), N\right)}{\mathbf{P}\left\{\xi_{1}+\xi_{2}+\ldots+\xi_{N}=m_{i}\left(\omega_{1}\right)\right\}} \\
& =\frac{B\left(m_{i}\left(\omega_{1}\right)\right)}{B\left(m_{i}\left(\omega_{1}\right), N\right)+\left(b_{2} C_{1}^{2} / 2 B(\theta)\right) B\left(m_{i}\left(\omega_{1}\right)-2, N-1\right) / N+C_{i}^{\prime} / N^{2}} \\
& =\left(1+\frac{1}{N} \frac{b_{2} C_{1}^{2}}{2 B(\theta)} \frac{m_{i}\left(\omega_{1}\right)\left(m_{i}\left(\omega_{1}\right)-1\right)}{1-m / N+2 / N} \frac{b_{0} B(\theta)}{\left(b_{1} C_{1}\right)^{2}}+\frac{1}{B\left(m_{i}\left(\omega_{1}\right), N\right)} \frac{C_{i}^{\prime}}{N^{2}}\right)^{-1},
\end{aligned}
$$

где $0<C_{i}^{\prime} \leqslant C_{5}$.

Так как

$$
\begin{aligned}
\frac{1}{B\left(m_{i}\left(\omega_{1}\right), N\right)} & =\frac{m_{i}\left(\omega_{1}\right)}{N} \frac{m_{i}\left(\omega_{1}\right)-1}{N-1} \ldots \frac{1}{N-m_{i}\left(\omega_{1}\right)+1}\left(\frac{N}{\lambda}\right)^{m_{i}\left(\omega_{1}\right)}\left(\frac{B(\theta)}{b_{0}}\right)^{N} \\
& =\frac{m_{i}\left(\omega_{1}\right) !}{\lambda^{m_{i}\left(\omega_{1}\right)}} \exp \left(-\sum_{k=1}^{m_{i}\left(\omega_{1}\right)-1} \ln \left(1-\frac{k}{N}\right)\right)\left(\frac{B(\theta)}{b_{0}}\right)^{N} \\
& <m_{i}\left(\omega_{1}\right) ! \lambda^{-m_{i}\left(\omega_{1}\right)} e^{m_{i}\left(\omega_{1}\right)\left(m_{i}\left(\omega_{1}\right)-1\right) /(2 N)}\left(\frac{B(\theta)}{b_{0}}\right)^{N}
\end{aligned}
$$


и при $N \rightarrow \infty$

$$
\left(\frac{B(\theta)}{b_{0}}\right)^{N} \rightarrow e^{\lambda},
$$

в силу (20), (21) и (22) существуют такие $n_{0} \in \mathbf{N}$ и $\varepsilon>0$, что

$$
\begin{aligned}
0 \leqslant \max _{1 \leqslant i \leqslant n} I_{\left\{m_{i}\left(\omega_{1}\right) \geqslant 2\right\}}\left(\frac{1}{N} \frac{m_{i}\left(\omega_{1}\right)\left(m_{i}\left(\omega_{1}\right)-1\right)}{2} \frac{1}{1-m\left(\omega_{1}\right) / N+2 / N} \frac{b_{0} b_{2}}{\left(b_{1}\right)^{2}}\right. \\
\left.+\frac{1}{B\left(m_{i}\left(\omega_{1}\right), N\right)} \frac{C_{i}^{\prime}}{N^{2}}\right)<1
\end{aligned}
$$

и

$$
\max _{1 \leqslant i \leqslant n} \frac{m_{i}\left(\omega_{1}\right)}{N}<1, \quad \max _{1 \leqslant i \leqslant n} \frac{m_{i}\left(\omega_{1}\right)\left(m_{i}\left(\omega_{1}\right)-1\right)}{N}<1
$$

при $N, n>n_{0},\left|\alpha-\alpha_{0}\right|<\varepsilon$. Тогда, используя неравенство $-x \leqslant \ln (1-x) \leqslant-x+x^{2} / 2$, справедливое для $0<x<1$, при $N, n>n_{0},\left|\alpha-\alpha_{0}\right|<\varepsilon$, получаем, что

$$
\begin{aligned}
& -\sum_{i=1}^{n} I_{\left\{m_{i}\left(\omega_{1}\right) \geqslant 2\right\}(} \frac{1}{N} \frac{m_{i}\left(\omega_{1}\right)\left(m_{i}\left(\omega_{1}\right)-1\right)}{2} \frac{1}{1-m_{i}\left(\omega_{1}\right) / N+2 / N} \frac{b_{0} b_{2}}{\left(b_{1}\right)^{2}} \\
& \left.+\frac{1}{B\left(m_{i}\left(\omega_{1}\right), N\right)} \frac{C_{i}^{\prime}}{N^{2}}\right) \\
& \leqslant \ln (\mathbf{P}(A)) \leqslant-\sum_{i=1}^{n} I_{\left\{m_{i}\left(\omega_{1}\right) \geqslant 2\right\}}\left(\frac{1}{N} \frac{m_{i}\left(\omega_{1}\right)\left(m_{i}\left(\omega_{1}\right)-1\right)}{2} \frac{1}{1-m_{i}\left(\omega_{1}\right) / N+2 N} \frac{b_{0} b_{2}}{\left(b_{1}\right)^{2}}\right. \\
& \left.+\frac{1}{B\left(m_{i}\left(\omega_{1}\right), N\right)} \frac{C_{i}^{\prime}}{N^{2}}\right)+\frac{1}{2} \sum_{i=1}^{n} I_{\left\{m_{i}\left(\omega_{1}\right) \geqslant 2\right\}}\left(\frac{1}{N} \frac{m_{i}\left(\omega_{1}\right)\left(m_{i}\left(\omega_{1}\right)-1\right)}{2}\right. \\
& \times \frac{1}{1-m_{i}\left(\omega_{1}\right) / N+2 / N} \frac{b_{0} b_{2}}{\left(b_{1}\right)^{2}}+\frac{C_{i}^{\prime}}{B\left(m_{i}\left(\omega_{1}\right), N\right)} \frac{N^{2}}{N^{2}} \cdot
\end{aligned}
$$

Следовательно,

$$
\begin{aligned}
-\left(\max _{1 \leqslant i \leqslant n}\right. & \left.\frac{1}{1-m_{i}\left(\omega_{1}\right) / N+2 / N}\right) \frac{1}{2} \frac{b_{0} b_{2}}{\left(b_{1}\right)^{2}} \frac{1}{N} \sum_{i=1}^{n} I_{\left\{m_{i}\left(\omega_{1}\right) \geqslant 2\right\}}\left(m_{i}\left(\omega_{1}\right)\left(m_{i}\left(\omega_{1}\right)-1\right)\right) \\
-\frac{C_{5}}{N^{2}} \sum_{i=1}^{n} \frac{1}{B\left(m_{i}\left(\omega_{1}\right), N\right)} & \\
\leqslant & \ln (\mathbf{P}(A)) \leqslant-\left(\min _{1 \leqslant i \leqslant n} \frac{1}{1-m_{i}\left(\omega_{1}\right) / N+2 / N}\right) \\
& \times \frac{1}{2} \frac{b_{0} b_{2}}{\left(b_{1}\right)^{2}}\left(\frac{1}{N} \sum_{i=1}^{n} I_{\left\{m_{i}\left(\omega_{1}\right) \geqslant 2\right\}}\left(m_{i}\left(\omega_{1}\right)\left(m_{i}\left(\omega_{1}\right)-1\right)\right)\right) \\
& \times\left(1-\frac{1}{2} \frac{1}{N}\left(\max _{1 \leqslant i \leqslant n} \frac{1}{1-m_{i}\left(\omega_{1}\right) / N+2 / N} \frac{m_{i}\left(\omega_{1}\right)\left(m_{i}\left(\omega_{1}\right)-1\right)}{2}\right) \frac{b_{0} b_{2}}{\left(b_{1}\right)^{2}}\right. \\
& \left.-\frac{1}{2} \frac{C_{5}}{N^{2}} \sum_{i=1}^{n} \frac{1}{B\left(m_{i}\left(\omega_{1}\right), N\right)}\right) .
\end{aligned}
$$


Из (24) получаем

$$
0<\frac{1}{N^{2}} \sum_{i=1}^{n} \frac{1}{B\left(m_{i}\left(\omega_{1}\right), N\right)} \leqslant \exp \left(\max _{1 \leqslant i \leqslant n} \frac{m_{i}\left(\omega_{1}\right)\left(m_{i}\left(\omega_{1}\right)-1\right)}{2 N}\right) \frac{1}{N^{2}} \sum_{i=1}^{n} m_{i}\left(\omega_{1}\right) ! \lambda^{-m_{i}\left(\omega_{1}\right)} .
$$

Поэтому в силу (18) и (22)

$$
\frac{1}{N^{2}} \sum_{i=1}^{n} \frac{1}{B\left(m_{i}\left(\omega_{1}\right), N\right)} \rightarrow 0
$$

если $n, N \rightarrow \infty$ так, что $\alpha \rightarrow \alpha_{0}$. Неравенство (26) с учетом (19), (21), (27), доказывает теорему.

Замечание 2. Если $m$ - такая пуассоновская случайная величина, что ее параметр $\lambda_{1}<\lambda$, то $\mathbf{E}_{1} m ! \lambda^{-m}<\infty$. Поэтому $m$ удовлетворяет условию теоремы 1 .

\section{3. Пуассоновский случай}

Если $\xi_{i}(\theta)$ - независимые одинаково распределенные пуассоновские случайные величины с параметром $\theta$ (случай $b_{i}=1, i=0,1,2 \ldots$ ), то событие $A_{i}\left(\omega_{1}\right)$ является событием схемы размещения $m_{i}\left(\omega_{1}\right)$ различимых частиц по $N$ ячейкам [3]. Следовательно, его вероятность равна

$$
\mathbf{P}\left(A_{i}\left(\omega_{1}\right)\right)=\left(1-\frac{1}{N}\right)\left(1-\frac{2}{N}\right) \ldots\left(1-\frac{m_{i}\left(\omega_{1}\right)-1}{N}\right) .
$$

Поэтому в этом случае справедлив аналог теоремы 1 с более слабыми условиями на $m$.

Теорема 2. Пусть $\mathbf{E}_{1} m^{2}<\infty$. Предположим, что $\xi_{i}(\theta)-$ независимые одинаково распределенные пуассоновские случайные величины с параметром $\theta$. Тогда, если $\alpha \rightarrow \alpha_{0}$ при $n, N \rightarrow \infty$, mo

$$
\mathbf{P}(A) \rightarrow \exp \left(-\frac{\alpha_{0}}{2} \mathbf{E} I_{\{m \geqslant 2\}} m(m-1)\right)
$$

почти наверное.

Доказательство. По лемме 2 найдется $\Omega^{\prime} \subset \Omega_{1}$ такое, что $\mathbf{P}_{1}\left(\Omega^{\prime}\right)=1$ и для всех $\omega_{1} \in \Omega^{\prime}$ выполнено (19). Пусть $\omega_{1} \subset \Omega^{\prime}$. Выберем такие $n_{0} \in \mathbf{N}$ и $\varepsilon>0$, что

$$
\max _{1 \leqslant i \leqslant n} \frac{m_{i}\left(\omega_{1}\right)}{N}<1
$$

при $N, n>n_{0},\left|\alpha-\alpha_{0}\right|<\varepsilon$. Тогда, используя неравенство

$$
-x<\ln (1-x)<-x+\frac{x^{2}}{2}, \quad 0<x<1,
$$

для оценки (28), при $N, n>n_{0},\left|\alpha-\alpha_{0}\right|<\varepsilon$, получаем, что

$$
-\sum_{i=1}^{n} \sum_{j=1}^{m_{i}\left(\omega_{1}\right)-1} \frac{j}{N} \leqslant \ln (\mathbf{P}(A)) \leqslant-\sum_{i=1}^{n} \sum_{j=1}^{m_{i}\left(\omega_{1}\right)-1} \frac{j}{N}+\frac{1}{2} \sum_{i=1}^{n} \sum_{j=1}^{m_{j}\left(\omega_{1}\right)-1}\left(\frac{j}{N}\right)^{2} .
$$


Поэтому

$$
\begin{aligned}
&-\frac{1}{2} \frac{1}{N} \sum_{i=1}^{n} I_{\left\{m_{i}\left(\omega_{1}\right) \geqslant 2\right\}} m_{i}\left(\omega_{1}\right)\left(m_{i}\left(\omega_{1}\right)-1\right) \leqslant \ln (\mathbf{P}(A)) \\
& \leqslant\left(-1+\frac{1}{2} \max _{1 \leqslant i \leqslant n} \frac{m_{i}\left(\omega_{1}\right)}{N}\right) \frac{1}{2} \frac{1}{N} \sum_{i=1}^{n} I_{\left\{m_{i}\left(\omega_{1}\right) \geqslant 2\right\}} m_{i}\left(\omega_{1}\right)\left(m_{i}\left(\omega_{1}\right)-1\right) .
\end{aligned}
$$

Из (30) следует (29).

Для случая, когда $m_{i}=m-$ постоянные величины, теорема 2 получена в [7].

\section{Список литературы}

1. Новиков Ф. А., Дискретная математика для программистов. Питер, Санкт-Петербург, 2004.

2. Колчин В. Ф., Один класс предельных теорем для условных распределений. Литовский матем. сб. (1968) 8, №1, 53-63.

3. Колчин В. Ф., Случайные графы. Физматлит, Москва, 2000.

4. Колчин А. В., Предельные теоремы для обобщенной схемы размещения. Дискретная математика (2003) 15, №4, 143-157.

5. Колчин А. В., Колчин В. Ф., О переходе распределений сумм независимых одинаково распределенных случайных величин с одной решетки на другую в обобщенной схеме размещения. Дискретная математика (2006) 18, №4, 113-127.

6. Колчин А. В., Колчин В. Ф., Переход с одной решетки на другую распределений сумм случайных величин, встречающихся в обобщенной схеме размещения. Дискретная математика (2007) 19, №3, 15-21.

7. Avkhadiev F. G., Chuprunov A. N., The probability of a successful allocation of ball groups by boxes. Lobachevskii J. Math. (2007) 25, 3-5.

Статья поступила 17.10.2008.

Переработанный вариант поступил 11.02.2009. 